\title{
A Spatial Impedance Controller for Robotic Manipulation
}

\author{
Ernest D. Fasse, Member, IEEE, and Jan F. Broenink, Member, IEEE
}

\begin{abstract}
Mechanical impedance is the dynamic generalization of stiffness, and determines interactive behavior by definition. Although the argument for explicitly controlling impedance is strong, impedance control has had only a modest impact on robotic manipulator control practice. This is due in part to the fact that it is difficult to select suitable impedances given tasks. A spatial impedance controller is presented that simplifies impedance selection. Impedance is characterized using "spatially affine" families of compliance and damping, which are characterized by nonspatial and spatial parameters. Nonspatial parameters are selected independently of configuration of the object with which the robot must interact. Spatial parameters depend on object configurations, but transform in an intuitive, well-defined way. Control laws corresponding to these compliance and damping families are derived assuming a commonly used robot model. While the compliance control law was implemented in simulation and on a real robot, this paper emphasizes the underlying theory.
\end{abstract}

Index Terms-Compliant motion, impedance control, manipulation, stiffness control.

\section{INTRODUCTION}

$\mathbf{R}$ OBOTIC manipulators are dynamic systems and interact with other dynamic systems such as mechanical objects, tools, other robots and humans. It is generally accepted that during interaction with another system a manipulator must somehow accommodate constraints imposed by the system [1]. Accommodation can be achieved by both passive means [2]-[4] and by active control [5]-[10]. The currently limited ability of robotic manipulators to interact effectively with other dynamic systems can be attributed in part to a failure to effectively control manipulator impedance. Although the argument for explicitly controlling impedance is strong, impedance control has had only a modest impact on robotic manipulator control practice due largely to two factors: First, selecting impedances suitable for specific tasks is difficult. Second, assuming that a suitable impedance has been selected, controlling (modulating) impedance is difficult. This paper considers primarily the spatial transformation of impedance,

Manuscript received January 6, 1995; revised February 19, 1996. The work of E. D. Fasse was supported by a NSF-NATO post-Doctoral Fellowship award RCD-9353 707 and by the Mechatronics Research Centre Twente. This paper was recommended for publication by Associate Editor A. De Luca and Editor S. Salcudean upon evaluation of the reviewers' comments.

E. D. Fasse was with the Control Laboratory, Electrical Engineering Department, University of Twente, 7500 AE Enschede, The Netherlands. He is now with the Aerospace and Mechanical Engineering Department, University of Arizona, Tucson, AZ 85721 USA.

J. F. Broenink is with the Control Laboratory, Electrical Engineering Department, University of Twente, 7500 AE Enschede, The Netherlands.

Publisher Item Identifier S 1042-296X(97)03820-2. which is part of the impedance selection problem. Explicit control laws are derived based on a particular robot model, but the resulting performance and robustness of these control laws is not investigated. Performance and robustness issues of impedance control and related interactive control schemes are addressed elsewhere [11]-[15].

Consider the problem of compliance selection. Compliance is commonly described as a static relation between endpoint configuration and effort using generalized coordinates. For a six degree-of-freedom manipulator, the configuration of the robot end-effector can be identified via a chart $\phi$ with generalized coordinates $\xi$. Commonly, three Cartesian coordinates represent translation; three angles (e.g., roll, pitch, and yaw) represent orientation, so that $\xi=\left(x, y, z, \theta_{r}, \theta_{p}, \theta_{y}\right)$. Associated with these coordinates are generalized velocities $\dot{\xi}$ and generalized forces $\Xi$. Compliance is then a static relation between $\xi$ and $\Xi$. The most common relation is linear

$$
\Xi=K_{\phi}\left(\xi-\xi_{v}\right)
$$

where $K_{\phi}$ is a stiffness matrix. Tuple $\xi_{v}$ is called the virtual equilibrium configuration (or more casually the virtual configuration). Parameters $K_{\phi}$ and $\xi_{v}$ describe the desired, instantaneous behavior of the end-effector. The configurationwrench behavior of this apparently simple relation is complex, so that in practice it is quite difficult to select compliance parameters. This is due in part to a lack of understanding of the behavioral consequences of changing the various parameters, as was pointed out clearly for the case of compliance by Lončarić in his study of passive compliance [16]-[18]. The stiffness matrix is usually parameterized in terms of its eigenvectors and eigenvalues. These parameters are selected depending on the task to be performed and on the configuration of some rigid body in the environment with which the robot interacts. The problem of selection would be simplified if some of the compliance parameters (e.g., the eigenvalues of $K_{\phi}$ ) could be chosen independently of the configuration of the rigid body in the environment.

Unfortunately, neither the eigenvalues nor the eigenvectors of $K_{\phi}$ can be chosen independently of the configuration of the rigid body in the environment. Compliance parameters are usually selected by introducing a "task frame" and an associated "task chart" $\psi$ with generalized coordinates $v=$ $\left(x^{\prime}, y^{\prime}, z^{\prime}, \theta_{r}^{\prime}, \theta_{p}^{\prime}, \theta_{y}^{\prime}\right)$. We then have $\xi=\phi \circ \psi^{-1}(v)$ and $\dot{v}=J \dot{\xi}$, where $J$ is a Jacobian matrix. Using chart $\psi$ the compliance map is

$$
\Upsilon=K_{\psi}\left(v-v_{v}\right)
$$


where $\Upsilon$ is the array of generalized forces using chart $\psi$ and $K_{\psi}$ is a stiffness matrix. Selection of $v_{v}$ and $K_{\psi}$ is assumed to be straightforward. The virtual equilibrium coordinates and stiffness with respect to chart $\phi$ are

$$
\begin{aligned}
\xi_{v} & =\phi \circ \psi^{-1}\left(v_{v}\right) \\
K_{\phi} & =J^{t} K_{\psi} J .
\end{aligned}
$$

If (3) and (4) are satisfied then the compliance relations of (1) and (2) generate the same configuration-wrench behavior for small displacements of the end-effector configuration from the virtual equilibrium configuration, as desired. Matrices $K_{\psi}$ and $K_{\phi}$ do not necessarily have the same eigenvalues. The eigenvalues of $K_{\phi}$ will in general depend on the configuration of the object with which the robot must interact.

This paper presents families of impedances with simpler, more intuitive spatial transformation properties. These families are parametrized by two kinds of parameters, spatial and nonspatial. If the spatial parameters and the robot end-effector configuration are transformed by corresponding rigid body transformations, then the resultant wrenches with respect to the end-effector are unchanged, even if the displacements of the end-effector configuration from the virtual equilibrium configuration are large. This property simplifies parameter selection. The compliance and damping families presented are superficially more complex than those of current impedance control, but the underlying physical behavior is in fact easier to understand.

The classical notation commonly used in the impedance control literature is poorly suited to describe finite rotations in particular and rigid body transformations in general. As a result it is difficult to simply describe rotational compliance and damping using classical notation. The notation used in this document is based on the notation of differential geometry commonly used in nonlinear control theory and modern mechanics. This notation has the additional advantage of being less ambiguous than the classical notation.

We begin, therefore, with an introduction of terminology and notation used in the description of the controller. Section II is a review of standard rigid body kinematics; complete presentations are given in robotics textbooks. After definition of concepts, Section III presents spatially affine compliance and damping families. Section IV derives corresponding control laws for a serial, rigid link manipulator. The derivation of these control laws is based on existing techniques described in the literature on compliance and impedance control, stabilization of mechanical control systems, navigation, and obstacle avoidance literature [6], [19]-[21], [8], [22], [23]. Section V presents a case study to show how impedance could be selected to execute complex, spatial tasks. Section VI looks at the generality of the compliance family.

\section{REPRESENTATION OF RIGID BoDIES}

The configuration of a rigid body can be represented by a frame, a quadruplet $\left(p, e_{1}, e_{2}, e_{3}\right)$, where $p$ in turn represents the translational position of the body and vectors $e_{1}, e_{2}$ and $e_{3}$ are a positively oriented, orthonormal triplet representing orientation (see Fig. 1). Configuration can be identified with

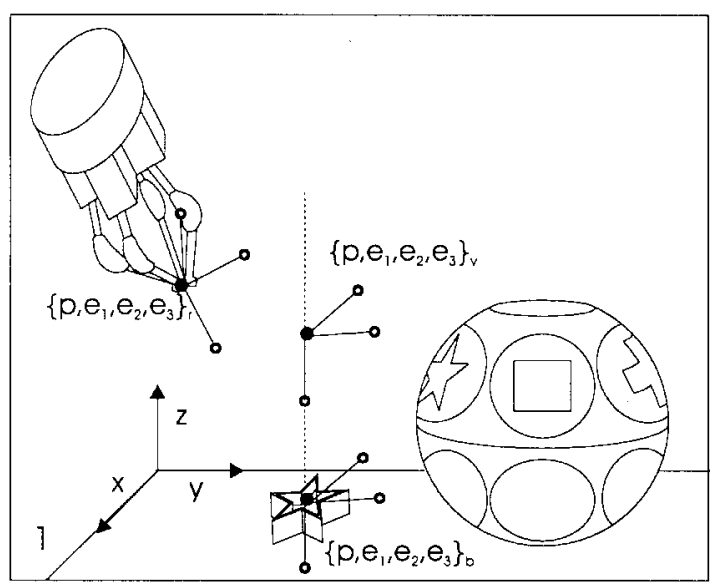

Fig. 1. Configurations of objects are represented by attached frames. Shown are frames representing the robot end-effector configuration, the virtual virtual equilibrium configuration, the block configuration and the hole configuration. Depicted differently is a stationary reference frame $x-y-z$.

a homogeneous matrix:

$$
H=\left[\begin{array}{ll}
R & p \\
0^{t} & 1
\end{array}\right]
$$

where $R=\left[\begin{array}{lll}e_{1} & e_{2} & e_{3}\end{array}\right]$ is a proper orthonormal matrix. Let $Q$ be the configuration manifold, so that $H \in Q$. Velocity, the temporal rate of change of configuration, is then a tangent vector $(H, \dot{H}) \in T Q$. We use the notation

$$
\dot{H}=\left[\begin{array}{cc}
\dot{R} & \dot{p} \\
0^{t} & 0
\end{array}\right]=\left[\begin{array}{cc}
R \tilde{\omega}_{b} & R v_{b} \\
0^{t} & 0
\end{array}\right]
$$

where

$$
\tilde{\omega}_{b}=\left[\begin{array}{ccc}
0 & -\omega_{3} & \omega_{2} \\
\omega_{3} & 0 & -\omega_{1} \\
-\omega_{2} & \omega_{1} & 0
\end{array}\right]
$$

is an antisymmetric matrix of angular speeds in the body frame corresponding to the array $\omega_{b}=\left[\begin{array}{lll}\omega_{1} & \omega_{2} & \omega_{3}\end{array}\right]^{t}$, and $v_{b}=\left[\begin{array}{lll}v_{1} & v_{2} & v_{3}\end{array}\right]^{t}$ is an array of translational speeds in the body frame. Infinitesimal displacements are denoted similarly.

Let $\sigma \in S E(3)$ be any rigid body transformation acting on $Q$. We do not distinguish notationally between group elements and their actions, writing

$$
\begin{aligned}
& \sigma: Q \rightarrow Q \\
& H \mapsto H_{\sigma} H
\end{aligned}
$$

where

$$
H_{\sigma}=\left[\begin{array}{cc}
R_{\sigma} & d_{\sigma} \\
0^{t} & 1
\end{array}\right]
$$

represents $\sigma$. The tangent action of $\sigma$ is a map from tangent velocities at one configuration to tangent velocities at a different configuration. Letting $H^{\prime}=H_{\sigma} H$ we have

$$
\begin{aligned}
& T \sigma: T Q \rightarrow T Q \\
& (H, \dot{H}) \mapsto\left(H^{\prime}, \dot{H}^{\prime}\right)
\end{aligned}
$$

where

$$
\dot{H}^{\prime}=\left[\begin{array}{cc}
R_{\sigma} R \tilde{\omega}_{b} & R_{\sigma} R v_{b} \\
0^{t} & 0
\end{array}\right]
$$


Geometrically, forces are covectors tangent to the configuration manifold, $(H, G) \in T^{*} Q$, and are energetically dual to velocities. We use the notation

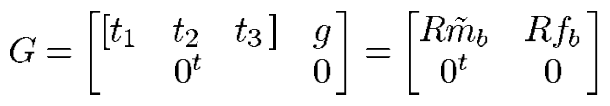

where $\tilde{m}_{b}$ is an antisymmetric matrix of torques in the body frame corresponding to the array of torques, $m_{b}$, and $f_{b}$ is an array of forces in the body frame. The projection of tangent forces onto wrenches (moments and forces) with respect to the body frame is denoted

$$
\begin{aligned}
\pi_{b}^{*}: T^{*} Q & \mapsto W_{b} \\
(H, G) & \mapsto\left[\begin{array}{cc}
\tilde{m}_{b} & f_{b} \\
0^{t} & 0
\end{array}\right]
\end{aligned}
$$

where $W_{b}$ can be identified with $s e^{*}(3)$. Forces and infinitesimal displacements can be paired to determine infinitesimal work. The infinitesimal work corresponding to force and infinitesimal displacement is

$$
\begin{aligned}
\delta W & =\langle(H, G),(H, \delta H)\rangle \\
& =f_{b}^{t} \delta p_{b}+\frac{1}{2} \operatorname{tr}\left(\tilde{m}_{b}^{t} \delta \tilde{\theta}_{b}\right) \\
& =f_{b}^{t} \delta p_{b}+m_{b}^{t} \delta \theta_{b}
\end{aligned}
$$

where $\operatorname{tr}(A)$ is the trace of matrix $A$. Forces and velocities can be paired similarly to determine instantaneous power.

\section{SPATIALly AfFine IMPEDANCE FAMILIES}

Choosing parameters of the family of impedances corresponding to (1) is difficult because the family does not have "desirable" spatial transformation properties. This section defines a "desirable" transformation property, spatial affinity. First, spatial affinity of compliance families is defined. Second, a particular affine compliance family is presented. Third, spatial affinity of damping families is defined. Fourth, a particular, spatially affine damping family is presented.

\section{A. Spatial Affinity of Compliance Families}

A family of compliances is a set of compliances indexed by sets of parameters. Each element of a compliance family $\left\{C_{s, n}\right\}$ is a conservative force field $C_{s, n}: Q \rightarrow T^{*} Q$. Index $s$ is an element of a set of parameters $S$ which is acted upon by group $S E(3)$; the action of rigid body transformation $\sigma \in S E(3)$ on $S$ is again denoted by $\sigma$. Index $n$ is an element of another set of parameters $N$ about which no special assumptions are made. Because the force field is conservative it is the differential of an energy function $C_{s, n}=d U_{s, n}$ where $U_{s, n}: Q \rightarrow \Re$. Compliance can also be thought of as being a map from configuration to body-equivalent wrenches $\pi_{b}^{*} \circ C_{s, n}: Q \longrightarrow W_{b}$.

Definition 1: A family of compliances, $\left\{C_{s, n}\right\}$, is spatially affine if $\pi_{b}^{*} \circ C_{\sigma(s), n} \circ \sigma=\pi_{b}^{*} \circ C_{s, n}$, independently of $s, n$, and $\sigma$. Set $S$ is then said to be a set of spatial parameters; set $N$ is then said to be a set of nonspatial parameters.

For a spatially affine family of compliances, if the spatial parameters and the configuration are transformed by corresponding rigid body transformations, then the resultant wrenches in any body frame are unchanged. This property simplifies compliance selection.

\section{B. A Spatial Compliance Family}

This subsection presents a particular, spatially affine compliance family. In the remainder of this paper such families shall be referred to as spatial compliance families. Elements of a family shall be referred to as spatial compliances.

Spatial compliance acts to align a frame attached to the robot end-effector $\left(p, e_{1}, e_{2}, e_{3}\right)_{r}$ to a virtual equilibrium frame $\left(p, e_{1}, e_{2}, e_{3}\right)_{v}$ as shown in Fig. 1 . The robot is made to perform useful tasks by moving this virtual frame, and by changing other compliance parameters. The virtual frame shall likely be moving continually, possibly into objects, making reaching any sort of equilibrium unlikely. Even if equilibrium is reached, the equilibrium configuration shall not likely be the virtual configuration due to interaction with other objects. Hence the term virtual equilibrium frame is used instead of equilibrium frame.

Let $R_{t}=\left[\begin{array}{lll}e_{1 t} & e_{2 t} & e_{3 t}\end{array}\right]$ be an orthonormal matrix. Let $\Gamma_{t}$ and $\Lambda_{o}$ be diagonal matrices. Let $K_{t}=R_{t} \Gamma_{t} R_{t}^{t}$. Let $s=\left(R_{t}, H_{v}\right)$ and $n=\left(\Gamma_{t}, \Lambda_{o}\right)$. The proposed family of spatial compliances is

$$
\begin{aligned}
& C_{s, n}: Q \rightarrow T^{*} Q \\
& {\left[\begin{array}{cc}
R_{r} & p_{r} \\
0^{t} & 1
\end{array}\right] \mapsto\left(\left[\begin{array}{cc}
R_{r} & p_{r} \\
0^{t} & 1
\end{array}\right],\left[\begin{array}{cc}
R_{r} \tilde{m}_{o} & K_{t} \Delta p \\
0^{t} & 0
\end{array}\right]\right)}
\end{aligned}
$$

where $\Delta p=p_{r}-p_{v}$ and

$$
\tilde{m}_{o}=\Lambda_{o} R_{v}^{t} R_{r}-R_{r}^{t} R_{v} \Lambda_{\circ} .
$$

The corresponding map from configuration to wrenches in the body frame is

$$
\begin{aligned}
& \pi_{b}^{*} \circ C_{s, n}: Q \rightarrow W_{b} \\
& \quad\left[\begin{array}{cc}
R_{r} & p_{r} \\
0^{t} & 1
\end{array}\right] \mapsto\left[\begin{array}{cc}
\tilde{m}_{o} & R_{r}^{t} K_{t} \Delta p \\
0^{t} & 0
\end{array}\right] .
\end{aligned}
$$

The compliance is more intuitively described in terms of its potential energy

$$
\begin{aligned}
& U_{s, n}: Q \rightarrow \Re \\
& H_{r} \mapsto \frac{1}{2} \Delta p^{t} K_{t} \Delta p-\operatorname{tr}\left(\Lambda_{o} R_{v}^{t} R_{r}\right) \\
&=\frac{1}{2} \Delta p^{t} K_{t} \Delta p-\sum_{i=1}^{3} \lambda_{i o} e_{i r}^{t} e_{i v}
\end{aligned}
$$

The first term is that of a translational spring of stiffness $K_{t}$, which acts to coincide points $p_{r}$ and $p_{v}$. The principal directions of stiffness are given by the orthonormal triplet $\left(e_{1 t}, e_{2 t}, e_{3 t}\right)$. The corresponding translational stiffnesses are $\left(\gamma_{1 t}, \gamma_{2 t}, \gamma_{3 t}\right)$. Each $-\lambda_{i o} e_{i r}^{t} e_{i v}$ term is the energy of an orientational compliance that acts to align vectors $e_{i r}$ and $e_{i v}$. The energy $-\lambda_{i o} e_{i r}^{t} e_{i v}$ is minimized when vectors $e_{i r}$ and $e_{i v}$ are aligned $\left(e_{i r}^{t} e_{i v}=1\right)$, and maximized when they are anti-aligned $\left(e_{i r}^{t} e_{i v}=-1\right)$. Parameters $\left(\lambda_{1 o}, \lambda_{2 o}, \lambda_{3 o}\right)$ are orientational costiffnesses. These parameters determine the orientational stiffnesses, as shall be shown. 
The spatial parameters of this family are the principal directions of translational stiffness, determined by $R_{t}$, and the virtual equilibrium configuration, $H_{v}$. The nonspatial parameters are the translational stiffnesses, $\Gamma_{t}$, and the orientational costiffnesses, $\Lambda_{\circ}$. The orientational potential energy function is similar to the navigation function on $S O(3)$ presented by Koditschek [21], who cites earlier work of Meyer [24].

To see that the force field of (15) is consistent with the energy function of (18), note that given an arbitrary infinitesimal displacement $\delta H_{r}$ from a given configuration $H_{r}$ the infinitesimal work $\delta W$ is

$$
\delta W=U_{s, n}\left(H_{r}+\delta H_{r}\right)-U_{s, n}\left(H_{r}\right)
$$

where

$$
H_{r}+\delta H_{r}=\left[\begin{array}{cc}
R_{r}+R_{r} \delta \tilde{\theta}_{b} & p_{r}+R_{r} \delta p_{b} \\
0^{t} & 1
\end{array}\right] .
$$

To first-order

$$
\begin{aligned}
\delta W & =\delta p_{b}^{t} R_{r}^{t} K_{t} \Delta p-\operatorname{tr}\left(\Lambda_{o} R_{v}^{t} R_{r} \delta \tilde{\theta}_{b}\right) \\
& =\delta p_{b}^{t} R_{r}^{t} K_{t} \Delta p+\frac{1}{2} \operatorname{tr}\left(\left(\Lambda_{o} R_{v}^{t} R_{r}-R_{r}^{t} R_{v} \Lambda_{o}\right)^{t} \delta \tilde{\theta}_{b}\right)
\end{aligned}
$$

because $\Lambda_{o}$ is symmetric and $\delta \tilde{\theta}_{b}$ is antisymmetric. From (14), $\delta W$ is equal to the infinitesimal work given $f_{b}=R_{r}^{t} K_{t} \Delta p$ and $\tilde{m}_{b}=\tilde{m}_{o}=\Lambda_{o} R_{v}^{t} R_{r}-R_{r}^{t} R_{v} \Lambda_{o}$, which is consistent with (15)-(17). A control law that corresponds to a desired $C_{s, n}$ is derived in Section IV. We now state two properties about this family, which are proven in the Appendix.

Claim 1: The family of compliances $\left\{C_{s, n}\right\}$ is spatially affine, i.e., $\pi_{b}^{*} \circ C_{\sigma(s), n} \circ \sigma=C_{s, n}$ for any parameters $s=\left(R_{t}, H_{v}\right)$ and $n=\left(\Gamma_{t}, \Lambda_{o}\right) ;$ and for any $\sigma \in S E(3)$.

Claim 2: Given strictly positive $\left(\gamma_{1 t}, \gamma_{2 t}, \gamma_{3 t}\right)$ and nonnegative, distinct $\left(\lambda_{1 o}, \lambda_{2 o}, \lambda_{3 o}\right)$, of which at least two are strictly positive, so that $\left(\lambda_{1 o}+\lambda_{20}\right),\left(\lambda_{2 o}+\lambda_{30}\right)$, and $\left(\lambda_{3 o}+\lambda_{1 o}\right)$ are strictly positive and $\left(\lambda_{1 o}^{2}-\lambda_{2 o}^{2}\right)\left(\lambda_{2 o}^{2}-\lambda_{3 o}^{2}\right)\left(\lambda_{3 o}^{2}-\lambda_{1 o}^{2}\right) \neq 0$; then for arbitrary $R_{t}$ and $H_{v}$ the corresponding energy map $U_{s, n}: Q \rightarrow \Re$ is a Morse function with four critical points, $\left\{H_{v}, H_{1}, H_{2}, H_{3}\right\}$, where

$$
H_{c}=\left[\begin{array}{cc}
R_{c} & p_{v} \\
0^{t} & 1
\end{array}\right]
$$

for

$$
\begin{aligned}
R_{c} \in & \left\{R_{v}, R_{1}, R_{2}, R_{3}\right\} \\
= & \left\{\left[\begin{array}{llll}
+e_{1 v} & +e_{2 v} & +e_{3 v}
\end{array}\right],\left[\begin{array}{llll}
+e_{1 v} & -e_{2 v} & -e_{3 v}
\end{array}\right],\right. \\
& {\left.\left[\begin{array}{llll}
-e_{1 v} & +e_{2 v} & -e_{3 v}
\end{array}\right],\left[\begin{array}{lll}
-e_{1 v} & -e_{2 v} & +e_{3 v}
\end{array}\right]\right\} . }
\end{aligned}
$$

Critical point $H_{v}$ is a global minimum of $U_{s, n}$ with a Morse index of six. The indexes of the remaining critical points are five, four, and three (not necessarily in that order).

\section{Spatial Affinity of Damping Families}

It is also possible to define the spatial affinity of a family of damping maps. Each element of a family of damping maps, $\left\{D_{\hat{s}, \hat{n}}\right\}$, is a map from velocity to force $D_{\hat{s}, \hat{n}}: T Q \rightarrow T^{*} Q$. Index $\hat{s}$ is an element of a set of parameters $\hat{S}$ which is acted upon by group $S E(3)$; index $\hat{n}$ is an element of another set of parameters, $\hat{N}$, about which no special assumptions are made. Damping can also be thought of as being a map from velocity to body-equivalent wrenches $\pi_{b}^{*} \circ D_{\hat{s}, \hat{n}}: T Q \rightarrow W_{b}$.

Definition 2: A family of damping maps, $\left\{D_{\hat{s}, \hat{n}}\right\}$, is spatially affine if $\pi_{b}^{*} \circ D_{\sigma(\hat{s}), \hat{n}} \circ T \sigma=\pi_{b}^{*} \circ D_{\hat{s}, \hat{n}}$, independently of $\hat{s}, \hat{n}$, and $\sigma$. Set $\hat{S}$ is then said to be a set of spatial parameters; set $\hat{N}$ is then said to be a set of nonspatial parameters.

\section{A Spatial Damping Family}

Spatial damping acts to retard motion between the robot end-effector frame $\left(p, e_{1}, e_{2}, e_{3}\right)_{r}$, and the virtual frame $\left(p, e_{1}, e_{2}, e_{3}\right)_{v}$. Let $\hat{R}_{t}=\left[\begin{array}{lll}\hat{e}_{1 t} & \hat{e}_{2 t} & \hat{e}_{3 t}\end{array}\right]$ be an orthonormal matrix. Let $\Gamma_{t}$ and $\hat{\Lambda}_{0}$ be diagonal matrices. Let $B_{t}=\hat{R}_{t} \hat{\Gamma}_{t} \hat{R}_{t}^{t}$. Let $\hat{s}=\left(\hat{R}_{t},(H, \dot{H})_{v}\right)$ and $\hat{n}=\left(\hat{\Gamma}_{t}, \hat{\Lambda}_{o}\right)$. Let in general

$$
\dot{H}_{\alpha}=\left[\begin{array}{cc}
R_{\alpha} \tilde{\omega}_{\alpha} & R_{\alpha} v_{\alpha} \\
0^{t} & 0
\end{array}\right]
$$

so that $\Delta \dot{p}=R_{r} v_{r}-R_{v} v_{v}$. A damping family compatible with the compliance family of (15) is

$$
\begin{aligned}
D_{\hat{s}, \hat{n}}: T Q & \rightarrow T^{*} Q \\
\left(H_{r}, \dot{H}_{r}\right) & \mapsto\left(\left[\begin{array}{cc}
R_{r} & p_{r} \\
0^{t} & 1
\end{array}\right],\left[\begin{array}{cc}
R_{r} \tilde{m}_{o}^{\prime} & B_{t} \Delta \dot{p} \\
0^{t} & 0
\end{array}\right]\right)
\end{aligned}
$$

where

$$
\tilde{m}_{o}^{\prime}=\left(\tilde{\omega}_{r}-\tilde{\omega}_{v}\right) \hat{\Lambda}_{o}+\hat{\Lambda}_{o}\left(\tilde{\omega}_{r}-\tilde{\omega}_{v}\right) .
$$

The corresponding map from velocity to wrenches in the body frame is

$$
\begin{aligned}
\pi_{b}^{*} \circ D_{\hat{s}, \hat{n}}: T Q \rightarrow W_{b} & \\
\left(H_{r}, \dot{H}_{r}\right) & \mapsto\left(\left[\begin{array}{cc}
R_{r} & p_{r} \\
0^{t} & 1
\end{array}\right],\left[\begin{array}{cc}
\tilde{m}_{o}^{\prime} & R_{r}^{t} B_{t} \Delta \dot{p} \\
0^{t} & 0
\end{array}\right]\right) .
\end{aligned}
$$

The translational term is that of a linear element of damping matrix $B_{t}$. The principal directions of damping are given by the orthonormal triplet $\left(\hat{e}_{1 t}, \hat{e}_{2 t}, \hat{e}_{3 t}\right)$. The corresponding damping coefficients are $\left(\hat{\gamma}_{1 t}, \hat{\gamma}_{2 t}, \hat{\gamma}_{3 t}\right)$. Parameters $\left(\hat{\lambda}_{1 o}, \hat{\lambda}_{2 o}, \hat{\lambda}_{3 o}\right)$ are orientational codamping coefficients. Orientational damping acts to resist rotation of $\left(e_{1 r}, e_{2 r}, e_{3 r}\right)$ with respect to $\left(e_{1 v}, e_{2 v}, e_{3 v}\right)$; coefficient $\hat{\lambda}_{i o}$ determines the resistance to rotation of $e_{i r}$ with respect to $e_{i v}$.

The spatial parameters of this damping family are the virtual velocity, $(H, \dot{H})_{v}$, and the principal directions of translational damping, $\hat{R}_{t}$. The nonspatial parameters are the translational damping coefficients, $\hat{\Gamma}_{t}$, and the orientational codamping coefficients, $\hat{\Lambda}_{o}$. The corresponding instantaneous power is

$$
P_{\text {damp }}=\Delta \dot{p}^{t} B_{t} \dot{p}_{r}-\frac{1}{2} \operatorname{tr}\left(\tilde{m}_{o}^{\prime} \tilde{\omega}_{r}\right) .
$$

A control law that corresponds to a desired $D_{\hat{s}, \hat{n}}$ is derived in Section IV. We next state two properties about this family, which are proven in the Appendix.

Claim 3: The family of damping maps $\left\{D_{\hat{s}, \hat{n}}\right\}$ is spatially affine, i.e., $\pi_{b}^{*} \circ D_{\sigma(\hat{s}), \hat{n}} \circ T \sigma=D_{\hat{s}, \hat{n}}$ for any parameters $s=\left(\hat{R}_{t},(H, \dot{H})_{v}\right)$ and $n=\left(\hat{\Gamma}_{t}, \hat{\Lambda}_{o}\right)$; and for any $\sigma \in S E(3)$. 
Claim 4: Given strictly positive $\left(\hat{\gamma}_{1 t}, \hat{\gamma}_{2 t}, \hat{\gamma}_{3 t}\right)$ and nonnegative $\left(\hat{\lambda}_{1 o}, \hat{\lambda}_{2 o}, \hat{\lambda}_{30}\right)$, of which at least two are strictly positive, so that $\left(\hat{\lambda}_{1 o}+\hat{\lambda}_{2 o}\right),\left(\hat{\lambda}_{2 o}+\hat{\lambda}_{3 o}\right)$ and $\left(\hat{\lambda}_{3 o}+\hat{\lambda}_{1 o}\right)$ are strictly positive; then for arbitrary $\hat{R}_{t}$ and $(H, \dot{H})_{v}$ with $\dot{H}_{v}=0$ the corresponding damping map $D_{\hat{s}, \hat{n}}$ is dissipative, i.e., $P_{\text {damp }}$ is a positive-definite function of $\dot{H}_{r}$.

If $D_{\hat{s}, \hat{n}}$ is chosen to be independent of $(H, \dot{H})_{v}$, the resulting map $D_{\hat{R}_{t}, \hat{n}}^{\prime}$ is dissipative and acts to resist rotation of the robot end-effector frame with respect to the stationary, inertial frame

$$
\begin{aligned}
D_{\hat{R}_{t}, \hat{n}}^{\prime}: T Q \rightarrow T^{*} Q \\
\left(H_{r}, \dot{H}_{r}\right) \mapsto\left(\left[\begin{array}{cc}
R_{r} & p_{r} \\
0^{t} & 1
\end{array}\right],\left[\begin{array}{cc}
R_{r}\left(\tilde{\omega}_{r} \hat{\Lambda}_{o}+\hat{\Lambda}_{o} \tilde{\omega}_{r}\right) & B_{t} R_{r} v_{r} \\
0^{t} & 0
\end{array}\right]\right) .
\end{aligned}
$$

\section{Robot Model and Derivation of CONTROL LaW}

In this section control laws are derived to achieve the desired spatial compliance and damping. The robot is assumed to be a serial linkage of rigid bodies with configuration manifold $\Theta$. Robot configuration is represented using a set of generalized coordinates that are measurable by sensors. For simplicity, the robot joints are assumed to be revolute; the coordinates are assumed to be joint angles. Robot configurations are related to end-effector configurations via the forward kinematic map, $L: \Theta \rightarrow Q$. Kinematically singular configurations are critical points of $L$, configurations at which some nonzero robot velocities are mapped onto zero end-effector velocities.

The dynamics of the robot are assumed to be dominated by: 1) inertia; 2) load-independent, configuration-dependent joint friction; and 3) gravity. The robot is actuated by a set of variable-effort actuators and interacts energetically with its environment via the end-effector. The efforts of the actuators are assumed to be the generalized forces corresponding to the generalized velocities. Here, actuator efforts are assumed to be joint torques. The dynamics of the robot are given by

$$
M(\theta) \ddot{\theta}+\tau_{c}(\theta, \dot{\theta})+\tau_{\mathrm{gr}}(\theta)+\tau_{\mathrm{fr}}(\theta, \dot{\theta})=\tau_{a}+\tau_{\mathrm{int}}
$$

where $M(\theta)$ is the inertia matrix, $\tau_{c}(\theta, \dot{\theta})$ are Coriolis and centripetal torques, $\tau_{g r}(\theta)$ are gravity torques, $\tau_{f r}(\theta, \dot{\theta})$ are friction torques, $\tau_{a}$ are the actuator torques, and $\tau_{\text {int }}$ are torques due to interaction with the environment via the end-effector. The sign convention is that given positive joint velocities: 1) the actuator and interaction torques are positive when work is being done on the robot; and 2) the gravity and friction torques are positive when negative work is done on the robot.

The overall structure of the control law is standard

$$
\begin{aligned}
\tau_{a}= & \tau_{-\mathrm{gr}}(\theta)+\tau_{\text {-fr }}(\theta, \dot{\theta}) \\
& -\tau_{\text {comp }}-\tau_{\text {damp }}(\theta, \dot{\theta})+\tau_{\mathrm{ff}}\left(\theta, \tau_{\mathrm{int}}\right)
\end{aligned}
$$

where $\tau_{-g r}(\theta)$ is a gravity compensation term, $\tau_{-f r}(\theta, \dot{\theta})$ is a friction compensation term, $\tau_{\text {comp }}$ is the spatial compliance term, and $\tau_{\text {damp }}$ is the spatial damping term. The remaining term $\tau_{f f}\left(\theta, \tau_{\text {int }}\right)$ is a force-feedback term, which is described in Section IV-C. The sign convention is that given positive joint velocities: 1) the gravity compensation, friction compensation, and force feedback torques are positive if work is done on the robot; and 2) the compliance and damping terms are positive if negative work is done on the robot.

\section{A. Spatial Compliance Control Law}

The derivation of the compliance control law is straightforward using techniques well founded in the literature on compliance and impedance control, stabilization of mechanical control systems, obstacle avoidance, and navigation [6], [19]-[21], [8], [22], [23]. Let $U_{\text {comp }}=U_{s, n} \circ L$ be the potential energy function of manipulator configuration $U_{\text {comp }}: \Theta \rightarrow \Re$. The control law is

$$
\begin{aligned}
\tau_{\mathrm{comp}} & =\frac{\partial U_{\mathrm{comp}}}{\partial \theta} \\
& =\left(\frac{\partial p_{r}}{\partial \theta}\right)^{t} K_{t}\left(p_{r}(\theta)-p_{v}\right)-\sum_{i=1}^{3} \lambda_{i o}\left(\frac{\partial e_{i r}}{\partial \theta}\right)^{t} e_{i v}
\end{aligned}
$$

This control law is based only on the kinestatic model of the robot. It fails to achieve the desired behavior at all kinematically singular configurations.

\section{B. Spatial Damping Control Law}

In deriving the control law corresponding to a desired damping map $D_{\hat{s}, \hat{n}}$ we use the fact that the instantaneous power is

$$
P_{\text {damp }}=\left(\dot{p}_{r}-\dot{p}_{v}\right)^{t} B_{t} \dot{p}_{r}+\sum_{i=1}^{3} \hat{\lambda}_{i o}\left(\dot{e}_{i r}^{t}-\dot{e}_{i v}^{t} R_{v} R_{r}^{t}\right) \dot{e}_{i r} .
$$

Substitution of $\dot{p}_{r}=\frac{\partial p_{r}}{\partial \theta} \dot{\theta}$ and $\dot{e}_{i r}=\frac{\partial e_{i r}}{\partial \theta} \dot{\theta}$ into (33) yields

$$
\begin{aligned}
P_{\text {damp }}= & \left(\left(\frac{\partial p_{r}}{\partial \theta}\right) \dot{\theta}-\dot{p}_{v}\right)^{t} B_{t} \frac{\partial p_{r}}{\partial \theta} \dot{\theta} \\
& +\sum_{i=1}^{3} \hat{\lambda}_{i o}\left(\dot{\theta}^{t}\left(\frac{\partial e_{i r}}{\partial \theta}\right)^{t}-\dot{e}_{i v}^{t} R_{v} R_{r}^{t}\right)\left(\frac{\partial e_{i r}}{\partial \theta}\right) \dot{\theta}
\end{aligned}
$$

We also have that $P_{\text {damp }}=\tau_{\text {damp }}^{t} \dot{\theta}$, which must be true for arbitrary $\dot{\theta}$, so that the control law is

$$
\begin{aligned}
\tau_{\text {damp }}= & \left(\frac{\partial p_{r}}{\partial \theta}\right)^{t} B_{t}\left(\left(\frac{\partial p_{r}}{\partial \theta}\right) \dot{\theta}-\dot{p}_{v}\right) \\
& +\sum_{i=1}^{3} \hat{\lambda}_{i o}\left(\frac{\partial e_{i r}}{\partial \theta}\right)^{t}\left(\left(\frac{\partial e_{i r}}{\partial \theta}\right) \dot{\theta}-R_{r} R_{v}^{t} \dot{e}_{i v}\right) .
\end{aligned}
$$

The control law corresponding to the dissipative map $D_{\hat{R}_{t}, \hat{n}}^{\prime}$ retarding motion with respect to the inertial frame is

$$
\tau_{\text {damp }}^{\prime}=\left(\frac{\partial p_{r}}{\partial \theta}\right)^{t} B_{t}\left(\frac{\partial p_{r}}{\partial \theta}\right) \dot{\theta}+\sum_{i=1}^{3} \hat{\lambda}_{i o}\left(\frac{\partial e_{i r}}{\partial \theta}\right)^{t}\left(\frac{\partial e_{i r}}{\partial \theta}\right) \dot{\theta}
$$

These control laws are, again, based only on the kinestatic model of the robot and fail to achieve the desired behavior at kinematically singular configurations. For quasistatic tasks, spatial affinity of damping is less important than spatial affinity of compliance, so that using a simpler damping control term may be warranted. A particularly simple alternative is linear, joint velocity feedback: $\tau_{\text {damp }}=B_{j} \dot{\theta}$, where $B_{j}$ is a strictly 
positive-definite, symmetric matrix of joint velocity feedback gains.

\section{Scaling Force Feedback Control Law}

It is well known that force feedback can be used to reduce the apparent inertia of a robot. It is less well known that force feedback can also be used to reduce the effects of uncompensated gravity and friction, which may be of more practical significance. Assume that the force feedback term of (31) is set to be a constant, scalar multiple of the interaction torque, $\tau_{\mathrm{ff}}=(\gamma-1) \tau_{\mathrm{int}}$. The interaction torque is not likely to be directly measurable, so that a suitable control law has to be derived. The interaction force $(H, G)_{\mathrm{int}} \in T^{*} Q$ is

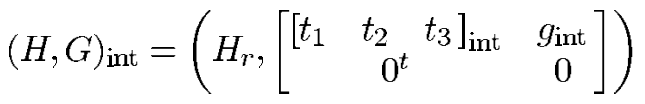

$$
\begin{aligned}
& =\left(H_{r},\left[\begin{array}{cc}
R_{r} \tilde{m}_{s} & R_{r} f_{s} \\
0^{t} & 0
\end{array}\right]\right)
\end{aligned}
$$

where the body-relative torques $\tilde{m}_{s}$ and forces $f_{s}$ are assumed to be measured by a sensor so that $(H, G)_{\mathrm{int}}$ is computable. Given velocity $(H, \dot{H})_{r} \in T Q$ and interaction force $(H, G)_{\mathrm{int}} \in T^{*} Q$, the power of interaction is

$$
\begin{aligned}
P_{\mathrm{int}} & =g_{\mathrm{int}}^{t} \dot{p}_{r}+\frac{1}{2} \sum_{i=1}^{3} t_{i \mathrm{int}} \dot{e}_{i r} \\
& =g_{\mathrm{int}}^{t} \frac{\partial p_{r}}{\partial \theta} \dot{\theta}+\frac{1}{2} \sum_{i=1}^{3} t_{i \mathrm{int}} \frac{\partial e_{i r}}{\partial \theta} \dot{\theta} .
\end{aligned}
$$

We also have that $P_{\mathrm{int}}=\tau_{\mathrm{int}}^{t} \dot{\theta}$, which must be true for arbitrary $\dot{\theta}$, so that

$$
\tau_{\mathrm{int}}=\left(\frac{\partial p_{r}}{\partial \theta}\right)^{t} g_{\mathrm{int}}+\frac{1}{2} \sum_{i=1}^{3}\left(\frac{\partial e_{i r}}{\partial \theta}\right)^{t} t_{i \mathrm{int}}
$$

when

$$
\tau_{f f}=(\gamma-1)\left(\frac{\partial p_{r}}{\partial \theta}\right)^{t} g_{\mathrm{int}}+\frac{\gamma-1}{2} \sum_{i=1}^{3}\left(\frac{\partial e_{i r}}{\partial \theta}\right)^{t} t_{i i n t}
$$

which is the desired control law. This is similar to, for example, Yoshikawa's $J_{y}^{t}\left(K_{d}-I\right) F$ term [25, eq. (6.30)]. Choosing $K_{d}=\gamma I$ ensures that the robot behave like a passive system. This approach is robust to modeling errors of robot inertia, gravity and friction. It is sensitive to modeling errors of robot kinematics, and to unmodeled actuator and force transducer dynamics. This limited the range of usable $\gamma^{\prime}$ s in experiments, in which $\gamma$ ranged between 1 and 2 .

\section{Coupled and Isolated Stability}

Controlled robots are sometimes stable when isolated, but unstable (e.g., exhibiting limit cycles) when coupled to other systems [12], [26]-[28], [15], [10]. This phenomenon is sometimes referred to as contact instability or coupled instability. Controlled robots that behave like passive systems are known to have robust coupled stability properties [12], [26], [15]. In

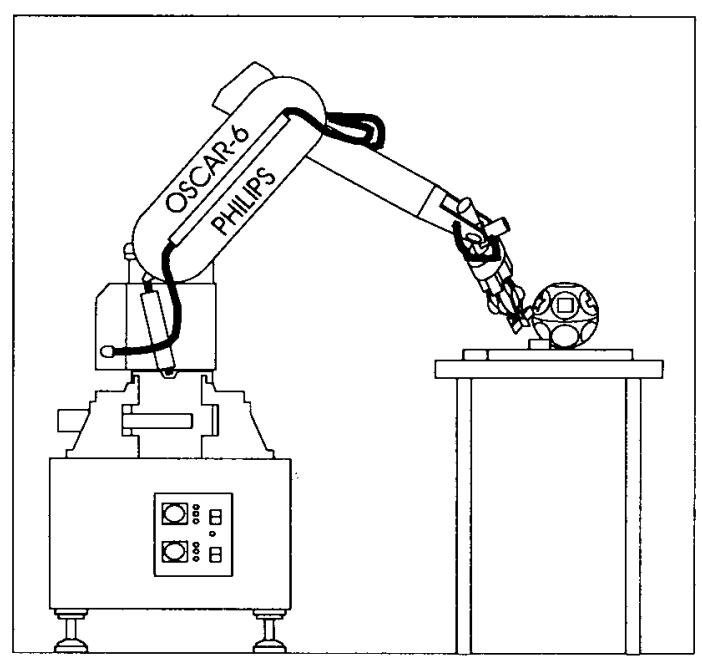

Fig. 2. The OSCAR-6 is a six degree-of-freedom, anthropomorphic robot. The hollebol sphere and blocks rest on a table.

practice, making robots behave like passive systems is difficult due to nonideal, perhaps unmodeled dynamics [12], [15].

Assume the following stationary impedance parameters to be given: 1) virtual configuration $H_{v}$ in the manipulator workspace, so that $\dot{H}_{v}=0$ and $L^{-1}\left(H_{v}\right)$ is nonempty; 2) strictly positive $\left(\gamma_{1 t}, \gamma_{2 t}, \gamma_{3 t}\right)$ and nonnegative, distinct $\left(\lambda_{1 o}, \lambda_{2 o}, \lambda_{3 o}\right)$, of which at least two are strictly positive; 3$)$ strictly positive $\left(\hat{\gamma}_{1 t}, \hat{\gamma}_{2 t}, \hat{\gamma}_{3 t}\right)$ and nonnegative $\left(\hat{\lambda}_{1 o}, \hat{\lambda}_{2 o}, \hat{\lambda}_{3 o}\right)$, of which at least two are strictly positive; and 4) strictly positive $\gamma$. For the case that $\dot{H}_{v}=0$ the damping control laws of (35) and (36) are equivalent. Given these assumptions the controlled robot is: 1) passive and 2) locally, asymptotically stable when isolated. Passivity is a consequence of Claims 2 and 4. Isolated stability is a consequence of Claims 2 and 4, and of [21, Proposition 3.6]. Detailed proofs are omitted for brevity. This is not surprising as the asymptotic stability properties of dissipative mechanical systems are well understood [21]-[23] and exploited in obstacle avoidance and navigation [19]-[21].

\section{Hollebol EXAMPLE}

In this section we look at an assembly task to make plausible the claim that the proposed control algorithms could be used to program a real robot to perform a useful task. In passing we draw some very simple conclusions based on the results of an experiment performed by Bonnes and Colard [29], Hoogeveen [30], and Tigchelaar [31]. As we draw only simple conclusions from their results, the experiment is not described in detail.

The work reported here was performed in context of the so-called Hollebol Project. The hollebol is a spatial puzzle for toddlers consisting of a plastic sphere with various ellipticaland polygonal-shaped holes, and blocks of corresponding shapes. The goal of the project was to assemble the hollebol using an anthropomorphic robot, a vision system, and a parallel computing system. The Philips OSCAR-6 robot is shown schematically in Fig. 2. A peg-tilting strategy [32]-[34] was chosen for assembly. This strategy can be broken up into a number of phases: rough alignment of the gripper with the 

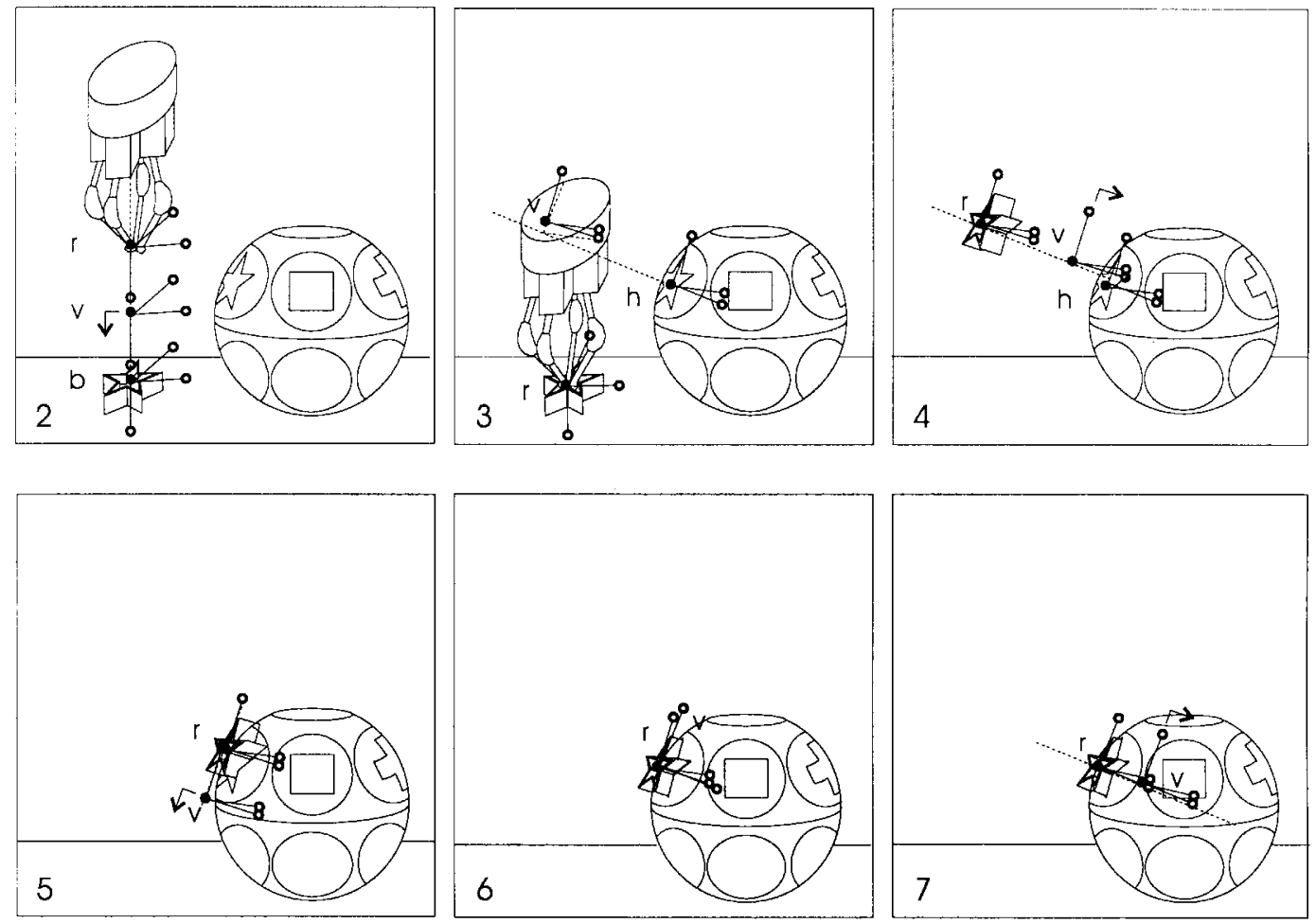

Fig. 3. The assembly task is decomposed into seven phases. (1) Rough alignment of the gripper with the block, shown in Fig. 1. (2) Final alignment of the gripper with the block, after which the block is grasped. (3) Rough alignment of the block with the hole. (4) Pushing a feature of the block into the hole. (5) Alignment of the block feature with the corresponding hole feature. (6) Alignment of the block and hole axes. (7) Pushing the block into the hole.

block, final alignment of the gripper with the block, rough alignment of the block with the hole, pushing a feature of the block into the hole, alignment of the block feature with the corresponding hole feature, reorientation of the block, and pushing the block into the hole (Figs. 1 and 3). For each of these phases an appropriate impedance, most importantly compliance, must be selected depending on the configuration of the blocks and the holes.

This task is given solely as an example of a realistic, complex task that involves interaction of a robotic manipulator with objects in its environment. Readers interested in selecting impedance to guarantee assembly are referred to literature specifically addressing that topic [2], [3], [35]-[37], [4]. The control law implemented was

$$
\begin{aligned}
\tau_{a}= & \tau_{-\mathrm{gr}}(\theta)+\tau_{-f r}(\theta, \dot{\theta})-B_{j} \dot{\theta} \\
& -\left(\frac{\partial p_{r}}{\partial \theta}\right)^{t} K_{t}\left(p_{r}(\theta)-p_{v}\right)+\sum_{i=1}^{3} \lambda_{i o}\left(\frac{\partial e_{i r}}{\partial \theta}\right)^{t} e_{i v} \\
& +(\gamma-1)\left(\frac{\partial p_{r}}{\partial \theta}\right)^{t} g_{\mathrm{int}}+\frac{\gamma-1}{2} \sum_{i=1}^{3}\left(\frac{\partial e_{i r}}{\partial \theta}\right)^{t} t_{i \mathrm{int}} .
\end{aligned}
$$

This control law and the required kinestatic computations were computed at a rate of $250 \mathrm{~Hz}$ using five $20 \mathrm{MHz}$, Inmos T800 transputers and the parallel programming language Occam. An additional 12 transputers were used for safety monitoring, signal conditioning, supervisory control, communication with the vision system, communication with the user interface, and communication with the lab computer network. This demonstrates the computational feasibility of the proposed algorithms. The spatial impedance controller was part of a hierarchical control system.

Compliance selection is simplified because the family of compliances is spatially affine. Nonspatial impedance parameters; i.e., the translational stiffnesses, the orientational costiffnesses and the scaling force feedback parameter; can be chosen for each phase independently of the configurations of the block and hole. They may depend on other factors such as the block shape or the block material. Here we assume simply that an appropriate set of nonspatial parameters is given for each phase of the task and for each block shape. In this case parameters were chosen based on simulation of robot behavior and experiment.

To illustrate how spatial parameters can be chosen we consider phases 4-6. Prior to phase 4 the block has been grasped by the robot. Frame $\left(p, e_{1}, e_{2}, e_{3}\right)_{r}$ has been chosen so that $p_{r}$ is at the desired center of compliance and so that in equilibrium axes $\left(e_{1}, e_{2}, e_{3}\right)_{r}$ correspond with the desired principal axes of orientational compliance. Intuitively, $e_{3 r}$ can be chosen normal to the plane of the block; $e_{1 r}$ then lies in the plane of the block and can be chosen in the direction of a salient feature such as a corner, or axis of symmetry. Vector $e_{2 r}$ completes the orthonormal triplet. A frame attached to the hole, $\left(p, e_{1}, e_{2}, e_{3}\right)_{h}$, is chosen similarly. Hole configuration is assumed to be priorly known or computable on-line from sensory information.

The result of phase 3 is that the block has been displaced from the center of the hole a distance $-h$ along axis $e_{3 h}$ and a distance $d$ along axis $e_{1 h}$; the block been rotated by an angle $\beta$ around axis $e_{2 h}$ with respect to the hole, so that 
$p_{r} \approx p_{h}-h e_{3 h}+d e_{1 h}$ and $R_{r} \approx R_{\beta} R_{h}$, where

$$
R_{\beta}=\left[\begin{array}{ccc}
\cos (\beta) & 0 & \sin (\beta) \\
0 & 1 & 0 \\
-\sin (\beta) & 0 & \cos (\beta)
\end{array}\right] .
$$

The goal of phase 4 is to push the salient feature of the block into the center of the hole. The principal directions of translational stiffness are aligned with the hole frame, so that $R_{t}=R_{h}$. The virtual equilibrium orientation is the hole orientation rotated by angle $\beta$ around the $e_{2 h}$ axis, $R_{v}=R_{\beta} R_{h}$. The virtual equilibrium position of the robot is moved along the hole axis $e_{3 h}$ at a constant speed $s_{4}$ until initial contact is detected, i.e., $p_{v}(t)=p_{h}-\left(h-s_{4} t\right) e_{3 h}+d e_{1 h}$ until measured force $f_{3 r}$ exceeds a threshold.

The goal of phase 5 is to align corresponding salient features of the block and hole. Again we assign $R_{t}=R_{h}$ and $R_{v}=R_{\beta} R_{h}$. The virtual equilibrium position of the robot is moved along the axis of the salient feature, $e_{1 h}$, at a constant speed, $s_{5}$, until tangential contact is detected, i.e., $p_{v}(t)=p_{h}+\left(d-s_{5} t\right) e_{1 h}+b e_{3 h}$ until tangential force $f_{1 r}$ exceeds a threshold. The constant $b>0$ ensures that normal contact be maintained. After tangential contact the block and hole centers should be nearly equal $p_{r} \approx p_{h}$.

The goal of phase 6 is to reorient the block so that it is aligned with the hole. Again we assign $R_{t}=R_{h}$. The virtual equilibrium configuration is changed abruptly to coincide with that of the hole, so that $p_{v}=p_{h}$ and $R_{v}=R_{h}$ until the configurations are acceptably similar. In phase 7 the block is pushed into the hole by moving the virtual equilibrium configuration along axis $e_{3 h}$. We make no claims about this strategy except that it is reasonable and that it can be implemented trivially in software. Implementing the same strategy using the compliance of (1) would require additional, explicit impedance transformations.

Using this strategy, Bonnes, Colard, and Tigchelaar programmed the OSCAR-6 robot so that it was able to grasp blocks placed flat on a table, and then insert them into both i) accessible holes of the hollebol attached to a sturdy base, and ii) holes with shapes and chamfers identical to those of the hollebol, accurately machined in a flat plate. Fig. 4 shows histories of the vertical component of $p_{r}$ and the normal component of $f_{r}$ during a successful assembly trial. Four events are indicated in the figure. Initial contact of the endeffector with the block occurs at time $t_{1}$. Initial contact of the block with the hole occurs at time $t_{2}$. Tangential contact of the block with the hole wall occurs at time $t_{3}$. The block is released at time $t_{4}$.

This shows that there were impedance parameter values for which the controlled robot did not exhibit any obviously unacceptable behavior such as limit cycling or wild movements. The experiment was too limited in scope to justify stronger statements about the dynamic performance of the controller. As stated, the experiment also demonstrated that the proposed control algorithms can be computed at acceptably high rates using available technology. Finally, it makes plausible the claim that the proposed control algorithms could be used to program real robots to perform useful tasks.

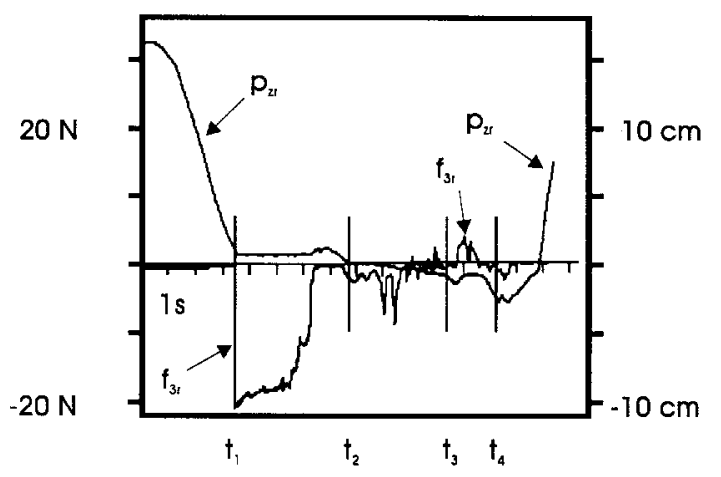

Fig. 4. History of $p_{z r}$ and $f_{3 r}$ during a successful assembly sequence.

\section{GENERALITY}

This concluding section looks at the generality of the family $\left\{C_{s, n}\right\}$, making use of known properties of compliantly supported rigid bodies for small displacements. For a compliantly supported, planar rigid body there exists a point called the center of compliance (center of flexure) at which for small displacements translational and rotational elasticity are decoupled [38]. Through the center of compliance there exist principal axes of translational compliance, along any one of which an applied force results in pure translation of the body along the axis; and principal axes of rotational compliance, about any one of which an applied torque results in pure rotation of the body about the axis. If the center of compliance lies outside the physical boundaries of the body it is known as a remote center of compliance. The center of compliance, the principal axes of translational compliance and the principal axes of rotational compliance are important parameters in the design of compliantly supported rigid bodies, including the well-known remote center compliance device [2], [4].

Lončarić [17], [18] showed that for a compliantly supported, spatial rigid body there does not always exist a point at which translational and rotational elasticity are decoupled, but that there does exist a point at which they are maximally decoupled, which again can be called a center of compliance. ${ }^{1}$ At an arbitrary point on the body, infinitesimal displacements and wrenches with respect to some body frame $\left(p, e_{1}, e_{2}, e_{3}\right)$ are related by a symmetric stiffness matrix:

$$
\left[\begin{array}{c}
R \delta m_{\text {comp }} \\
R \delta f_{\text {comp }}
\end{array}\right]=K\left[\begin{array}{l}
R \delta \theta_{b} \\
R \delta p_{b}
\end{array}\right]=\left[\begin{array}{ll}
K_{t} & K_{c} \\
K_{c} & K_{o}
\end{array}\right]\left[\begin{array}{l}
R \delta \theta_{b} \\
R \delta p_{b}
\end{array}\right] .
$$

The set of symmetric, $6 \times 6$ matrices is 21 -dimensional. Any symmetric matrix can be expressed in the form $K=R \Gamma R^{t}$, where $R$ is an element of the 15-dimensional set $S O(6)$ and $\Gamma$ is an element of the 6-dimensional set of diagonal, $6 \times$ 6 matrices. Matrices $K_{t}$ and $K_{o}$ are necessarily symmetric at any point on the body. If $\operatorname{tr}\left(K_{t}\right)$ is not an eigenvalue of $K_{t}$, then matrix $K_{c}$ is symmetric at a unique point, the center of compliance. To approximate arbitrary compliances

${ }^{1}$ Lončarić used the term center of stiffness. In this paper we use the a-causal definition that a compliant element is any nonrigid element for which there is a relationship between the displacement and effort of the element. A causal definition of a compliant element is that the displacement of the element is a function of the externally imposed effort. The corresponding causal definition of a stiff element is that the effort exerted by the element is a function of the externally imposed displacement. 
locally, a family of compliances should have a 21-dimensional parameter space, excluding the virtual equilibrium configuration parameter: three dimensions are necessary to specify the location of the center of compliance, 12 are necessary to specify $K_{t}$ and $K_{o}$, and 6 are necessary to specify $K_{c}$.

The parameter space of family $\left\{C_{s, n}\right\}$, excluding the virtual equilibrium configuration parameter, is $S O(3) \times \Re^{3} \times \Re^{3}$, which is 9-dimensional. Parameters $R_{t}$ and $\Gamma_{t}$ can be used to specify an arbitrary $K_{t}$. Parameter $\Lambda_{0}$ specifies indirectly the eigenvalues of $K_{o}$, but not its eigenvectors. The only way to change the principal axes of rotational compliance and the center of compliance is to redefine $H_{r}$. This suggests that the compliance family needs to be generalized by letting the center of compliance $H_{c c}$ be different than $H_{r}$, i.e., by setting $H_{c c}=H_{r} H_{\varsigma}$. This would add six more parameter dimensions, resulting in a total of 15 . This would still be insufficiently general, as there would be no means to change $K_{c}$ at the center of compliance.

This begs the question: how should family $\left\{C_{s, n}\right\}$ be generalized so as to account for coupling between translational and rotational compliance at the center of compliance? Looking at the linear problem suggests a direction for future research. Assuming that $K_{c}$ is diagonal, the associated potential function for small displacements is $U_{c}=\alpha_{1} \delta \theta_{1} \delta p_{1}+\alpha_{2} \delta \theta_{2} \delta p_{2}+$ $\alpha_{3} \delta \theta_{3} \delta p_{3}$. This suggests that an appropriate, nonlinear potential function for large displacements be of the form

$U_{c}=\alpha_{1} \sin \left(\Delta \theta_{1}\right) \Delta p_{1}+\alpha_{2} \sin \left(\Delta \theta_{2}\right) \Delta p_{2}+\alpha_{3} \sin \left(\Delta \theta_{3}\right) \Delta p_{3}$

where the $\Delta \theta_{i}$ 's and the $\Delta p_{i}$ 's are measures of displacement of the robot frame from the virtual equilibrium frame. This is merely an indication of direction for future investigation and not a result. ${ }^{2}$

\section{APPENDIX \\ PROOFS OF Claims}

\section{Proof of Claim 1}

Given any $\sigma \in S E(3)$ represented by matrix $H_{\sigma}$ one computes $\pi_{b}^{*} \circ C_{\sigma(s), n} \circ \sigma$ straightforwardly in (45) as shown at the bottom of the page.

$$
\begin{aligned}
& C_{\sigma(s), n} \circ \sigma: Q \rightarrow T^{*} Q \\
& H_{r} \mapsto\left(H_{\sigma} H_{r},\right. \\
& \left.\left[\begin{array}{cc}
R_{\sigma} R_{r}\left(\Lambda_{O} R_{v}^{t} R_{r}-R_{r}^{t} R_{v} \Lambda_{o}\right) & R_{\sigma} R_{t} \Gamma_{t} R_{t}^{t}\left(p_{r}-p_{v}\right) \\
0^{t} & 0
\end{array}\right]\right) \\
& \quad \pi_{b}^{*} \circ C_{\sigma(s), n} \circ \sigma: Q \longrightarrow W_{b} \\
& H_{r} \mapsto\left[\begin{array}{cc}
\Lambda_{o} R_{v}^{t} R_{r}-R_{r}^{t} R_{v} \Lambda_{o} & R_{r}^{t} K_{t}\left(p_{r}-p_{v}\right) \\
0^{t} & 0
\end{array}\right] .
\end{aligned}
$$

\footnotetext{
${ }^{2}$ This generalization has since been largely completed.
}

Comparing this with (16) and (17) we see that $\pi_{b}^{*} \circ C_{\sigma(s), n} \circ \sigma=$ $C_{s, n}$, proving Claim 1 .

\section{Proof of Claim 2}

The proof of Claim 2 is based on a lemma similar to Lemma 4.1 of Koditschek [21], which is derived from results of Chillingworth et al. [39]. Let $\left\{I, E_{1}, E_{2}, E_{3}\right\}$ be the set of diagonal, proper rotation matrices:

$$
\begin{aligned}
\left\{I, E_{1}, E_{2}, E_{3}\right\}=\left\{\left[\begin{array}{ccc}
+1 & 0 & 0 \\
0 & +1 & 0 \\
0 & 0 & +1
\end{array}\right],\left[\begin{array}{ccc}
+1 & 0 & 0 \\
0 & -1 & 0 \\
0 & 0 & -1
\end{array}\right],\right. \\
{\left.\left[\begin{array}{ccc}
-1 & 0 & 0 \\
0 & +1 & 0 \\
0 & 0 & -1
\end{array}\right],\left[\begin{array}{ccc}
-1 & 0 & 0 \\
0 & -1 & 0 \\
0 & 0 & +1
\end{array}\right]\right\} . }
\end{aligned}
$$

Lemma 1: Given $\Lambda_{o}$ with diagonal elements $\left(\lambda_{1 \circ}, \lambda_{2 o}, \lambda_{3 \circ}\right.$ such that $\left(\lambda_{1 \circ}^{2}-\lambda_{2 o}^{2}\right)\left(\lambda_{2 o}^{2}-\lambda_{3 o}^{2}\right)\left(\lambda_{3 o}^{2}-\lambda_{1 o}^{2}\right) \neq 0$, then for $R \in S O(3), \Lambda_{o} R-R^{t} \Lambda_{o}=0$ if and only if $R$ is one of the the four diagonal, proper rotation matrices:

$$
R=\left[\begin{array}{lll}
r_{11} & r_{12} & r_{13} \\
r_{21} & r_{22} & r_{23} \\
r_{31} & r_{32} & r_{33}
\end{array}\right] \in\left\{I, E_{1}, E_{2}, E_{3}\right\}
$$

Proof: $\Lambda_{o} R-R^{t} \Lambda_{o}=0$ if and only if $R \Lambda_{o} R=$ $\Lambda_{0}$, which is symmetric. Therefore $R^{t} \Lambda_{0} R^{t}=\Lambda_{0}$ and $R^{t} \Lambda_{o} R^{t} R \Lambda_{o} R=R^{t} \Lambda_{O}^{2} R=\Lambda_{o}^{2}$, implying that $\Lambda_{o}^{2} R-R \Lambda_{o}^{2}=$ 0 . Evaluation yields

$$
\left[\begin{array}{ccc}
0 & \left(\lambda_{1 o}^{2}-\lambda_{2 o}^{2}\right) r_{12} & \left(\lambda_{1 o}^{2}-\lambda_{3 o}^{2}\right) r_{13} \\
\left(\lambda_{2 o}^{2}-\lambda_{1 o}^{2}\right) r_{21} & 0 & \left(\lambda_{2 o}^{2}-\lambda_{3 o}^{2}\right) r_{23} \\
\left(\lambda_{3 o}^{2}-\lambda_{1 o}^{2}\right) r_{31} & \left(\lambda_{3 o}^{2}-\lambda_{2 o}^{2}\right) r_{32} & 0
\end{array}\right]=0 .
$$

Given that $\left(\lambda_{1 o}^{2}-\lambda_{2 o}^{2}\right)\left(\lambda_{2 o}^{2}-\lambda_{3 o}^{2}\right)\left(\lambda_{3 o}^{2}-\lambda_{1 o}^{2}\right) \neq 0$, this is true if and only if all off-diagonal elements of $R$ are zero, proving the lemma. If the assumption is relaxed additional solutions exist. For example, if $\lambda_{1 o}=\lambda_{2 o}$ then

$$
R(\theta)=\left[\begin{array}{ccc}
\cos (\theta) & -\sin (\theta) & 0 \\
-\sin (\theta) & -\cos (\theta) & 0 \\
0 & 0 & -1
\end{array}\right]
$$

is a solution for any $\theta$.

Moving on to the proof of Claim 2, a critical point $H_{c}$ of $U_{s, n}$ is a point for which the compliant force is zero, i.e., for which $d U_{s, n}\left(H_{c}\right)=C_{s, n}\left(H_{c}\right)=0$. Because the translational stiffnesses $\left(\gamma_{1 t}, \gamma_{2 t}, \gamma_{3 t}\right)$ are all nonzero, $K_{t}$ is nonsingular for any $R_{t}$, so that $p_{c}=p_{v}$ for any critical point. Apply the lemma to show that there are four critical points, substituting $R_{v}^{t} R_{r}$ for $R$. By assumption $\left(\lambda_{1 \circ}^{2}-\lambda_{2 o}^{2}\right)\left(\lambda_{2 o}^{2}-\lambda_{3 \circ}^{2}\right)\left(\lambda_{3 \circ}^{2}-\right.$ $\left.\lambda_{10}^{2}\right) \neq 0$. It follows that there are four rotation matrices [those given in (23)] at which $\Lambda_{o} R_{v}^{t} R_{r}$ is symmetric, and thus $R_{r}\left(\Lambda_{o} R_{v}^{t} R_{r}-R_{r}^{t} R_{v} \Lambda_{o}\right)$ is zero. From this we conclude that $\left\{H_{v}, H_{1}, H_{2}, H_{3}\right\}$ is the set of critical points of $U_{s, n}$.

$$
C_{s, n} \circ \sigma: Q \rightarrow T^{*} Q \text { such that } H_{r} \mapsto\left(H_{\sigma} H_{r}\left[\begin{array}{cc}
R_{\sigma} R_{r}\left(\Lambda_{o} R_{v}^{t} R_{\sigma} R_{r}-R_{r}^{t} R_{\sigma}^{t} R_{v} \Lambda_{o}\right) & K_{t}\left(R_{\sigma} p_{r}+d_{\sigma}-p_{v}\right) \\
0^{t} & 0
\end{array}\right]\right)
$$


A function $f$ is a Morse function if its second differential, $d^{2} f$, is nondegenerate at all of its critical points. One can compute the second differential of $U_{s, n}$ by linearizing $\pi_{b}^{*} \circ C_{s, n}$ about each of the four critical points, and then computing the corresponding stiffness matrix (Hessian matrix) relating infinitesimal displacements and wrenches, $\left(\delta \theta_{b}, \delta p_{b}\right)$ and $\left(\delta m_{\text {comp }}, \delta f_{\text {comp }}\right)$. Linearizing $\pi_{b}^{*} \circ C_{s, n}$ at $H_{v}$ one finds that

$$
\left[\begin{array}{c}
\delta m_{\text {comp }} \\
\delta f_{\text {comp }}
\end{array}\right] \approx\left[\begin{array}{cc}
\Gamma_{o} & 0 \\
0 & R_{v}^{t} K_{t} R_{v}
\end{array}\right]\left[\begin{array}{l}
\delta \theta_{b} \\
\delta p_{b}
\end{array}\right]
$$

where

$$
\Gamma_{o}=\left[\begin{array}{ccc}
\lambda_{2 o}+\lambda_{3 o} & 0 & 0 \\
0 & \lambda_{3 o}+\lambda_{1 o} & 0 \\
0 & 0 & \lambda_{1 o}+\lambda_{2 o}
\end{array}\right] .
$$

It follows that

$$
\left[\begin{array}{c}
R_{v} \delta m_{\mathrm{comp}} \\
R_{v} \delta f_{\mathrm{comp}}
\end{array}\right] \approx\left[\begin{array}{cc}
R_{v} \Gamma_{o} R_{v}^{t} & 0 \\
0 & R_{t} \Gamma_{t} R_{t}^{t}
\end{array}\right]\left[\begin{array}{l}
R_{v} \delta \theta_{b} \\
R_{v} \delta p_{b}
\end{array}\right]
$$

which determines the stiffness matrix. The Morse index is equal to the number of positive eigenvalues of the stiffness matrix, in this case six. This also shows that for $H_{r}$ near $H_{v}$, $\gamma_{10}=\lambda_{2 o}+\lambda_{3 o}$ is the effective rotational stiffness about axis $e_{1 v}, \gamma_{2 o}=\lambda_{3 o}+\lambda_{1 o}$ is the effective rotational stiffness about axis $e_{2 v}$, and $\gamma_{3 o}=\lambda_{1 o}+\lambda_{2 o}$ is the effective rotational stiffness about axis $e_{3 v}$. For $H_{r}$ near $H_{1}$ one finds that

$$
\left[\begin{array}{c}
R_{1} \delta m_{\text {comp }} \\
R_{1} \delta f_{\text {comp }}
\end{array}\right] \approx\left[\begin{array}{cc}
R_{1} \Gamma_{1} R_{1}^{t} & 0 \\
0 & R_{t} \Gamma_{t} R_{t}^{t}
\end{array}\right]\left[\begin{array}{l}
R_{1} \delta \theta_{b} \\
R_{1} \delta p_{b}
\end{array}\right]
$$

where

$$
\Gamma_{1}=\left[\begin{array}{ccc}
-\lambda_{2 o}-\lambda_{3 o} & 0 & 0 \\
0 & -\lambda_{3 o}+\lambda_{1 o} & 0 \\
0 & 0 & \lambda_{1 o}-\lambda_{2 o}
\end{array}\right] .
$$

Assume without loss of generality that $\lambda_{10}>\lambda_{2 o}>\lambda_{30}$; then the stiffness matrix has five positive eigenvalues, so that the Morse index at $H_{1}$ is five. The Morse indexes at $H_{2}$ and $H_{3}$ can be shown to be four and three, respectively. Furthermore, $-\operatorname{tr}\left(\Lambda_{o} R_{v}^{t} R_{r}\right)$ is a Morse function on the compact manifold $S O(3)$, and thus must have a global minimum at one or more critical points. Evaluation at the critical points shows that $R_{v}$ is a global minimum of $-\operatorname{tr}\left(\Lambda_{0} R_{v}^{t} R_{r}\right)$, whence $H_{v}$ is a global minimum of $U_{s, n}$, proving Claim 2.

\section{Proof of Claim 3}

Given any $\sigma \in S E(3)$ represented by matrix $H_{\sigma}$ one computes $\pi_{b}^{*} \circ D_{\sigma(\hat{s}), \hat{n}} \circ T \sigma$ straightforwardly as follows:

$$
\begin{aligned}
& D_{\hat{s}, \hat{n}} \circ T \sigma: T Q \rightarrow T^{*} Q \\
& (H, \dot{H})_{r} \mapsto\left(H_{\sigma} H_{r},\right. \\
& \left.\left[\begin{array}{cc}
R_{\sigma} R_{r}\left(\Delta \tilde{\omega} \hat{\Lambda}_{o}+\hat{\Lambda}_{o} \Delta \tilde{\omega}\right) & \hat{R}_{t} \hat{\Gamma}_{t} \hat{R}_{t}^{t}\left(R_{\sigma} R_{r} v_{r}-R_{v} v_{v}\right) \\
0^{t} & 0
\end{array}\right]\right)
\end{aligned}
$$

where $\Delta \tilde{\omega}=\tilde{\omega}_{r}-\tilde{\omega}_{v}$. Thus

$$
\begin{aligned}
& D_{\sigma(\hat{s}), \hat{n}} \circ T \sigma: T Q \rightarrow T^{*} Q \\
& (H, \dot{H})_{r} \mapsto\left(H_{\sigma} H_{r},\right. \\
& \left.\left[\begin{array}{cc}
R_{\sigma} R_{r}\left(\Delta \tilde{\omega} \hat{\Lambda}_{o}+\hat{\Lambda}_{o} \Delta \tilde{\omega}\right) & R_{\sigma} \hat{R}_{t} \hat{\Gamma}_{t} \hat{R}_{t}^{t}\left(R_{r} v_{r}-R_{v} v_{v}\right) \\
0^{t} & 0
\end{array}\right]\right)_{(58)} \\
& \quad \pi_{b}^{*} \circ D_{\sigma(\hat{s}), \hat{n} \circ T \sigma: T Q \rightarrow W_{b}} \\
& \quad(H, \dot{H})_{r} \mapsto\left[\begin{array}{cc}
\left(\Delta \tilde{\omega} \hat{\Lambda}_{o}+\hat{\Lambda}_{o} \Delta \tilde{\omega}\right) & R_{r}^{t} B_{t} \Delta \dot{p} \\
0^{t} & 0
\end{array}\right]
\end{aligned}
$$

Comparing this with (26) and (27) we see that $\pi_{b}^{*} \circ D_{\sigma(\hat{s}), \hat{n}} \circ$ $T \sigma=D_{\hat{s}, \hat{n}}$, proving Claim 3 .

\section{Proof of Claim 4}

The instantaneous power given by (28) is equivalent to

$$
\begin{aligned}
P_{\text {damp }}= & \Delta \dot{p}^{t} B_{t} \dot{p}_{r}+\left(\hat{\lambda}_{2 o}+\hat{\lambda}_{3 o}\right) \omega_{1 r}\left(\omega_{1 r}-\omega_{1 v}\right) \\
& +\left(\hat{\lambda}_{3 o}+\hat{\lambda}_{1 o}\right) \omega_{2 r}\left(\omega_{2 r}-\omega_{2 v}\right) \\
& +\left(\hat{\lambda}_{1 o}+\hat{\lambda}_{2 o}\right) \omega_{3 r}\left(\omega_{3 r}-\omega_{3 v}\right) .
\end{aligned}
$$

For $\dot{H}_{v}=0$ this is equivalent to

$$
\begin{aligned}
P_{\text {damp }}= & \dot{p}_{r}^{t} B_{t} \dot{p}_{r}+\left(\hat{\lambda}_{2 o}+\hat{\lambda}_{3 o}\right) \omega_{1 r}^{2} \\
& +\left(\hat{\lambda}_{3 o}+\hat{\lambda}_{1 o}\right) \omega_{2 r}^{2}+\left(\hat{\lambda}_{1 o}+\hat{\lambda}_{2 o}\right) \omega_{3 r}^{2}
\end{aligned}
$$

which is a positive-definite function of $\dot{H}_{r}$ because $B_{t}=$ $\hat{R}_{t} \hat{\Gamma}_{t} \hat{R}_{t}^{t}$ has strictly positive eigenvalues. This proves Claim 4 .

\section{ACKNOWLEDGMENT}

The Hollebol project was performed in cooperation with the Laboratory for Measurement and Instrumentation. The authors would like to thank the many participants in the Hollebol project. They are grateful for the scientific cooperation of $\mathrm{J}$. van Amerongen, M. J. Korsten, and M. J. L. Tiernego; and for the technical cooperation of R. Bruis, H. Roebbers, and M. H. Schwirtz. Special thanks go to the "OSCAR-boys," A. J. Bonnes, G. J. B. Colard, and T. Tigchelaar, who designed and implemented the control system for the OSCAR-6 robot in a very short time. They would like to thank N. Hogan, B. M. J. Maschke, S. Stramigioli, and the anonymous reviewers for their comments on the manuscript.

\section{REFERENCES}

[1] R. P. C. Paul and B. Shimano, "Compliance and control," in Proc. Joint Automatic Control Conf., 1976, pp. 694-699.

[2] S. H. Drake, "Using compliance in lieu of sensory feedback for automatic assembly," Ph.D. dissertation, Massachusetts Institute of Technology, Cambridge, 1977.

[3] M. T. Mason, "Compliance and force control for computer-controlled manipulators," IEEE Trans. Syst., Man Cybern., vol. SMC-11, pp. 418-432, 1981

[4] D. Whitney, "Quasi-static assembly of compliantly supported rigid parts," ASME J. Dyn. Syst., Measure. Contr., vol. 104, pp. 65-77, 1982.

[5] N. Hogan, "Mechanical impedance control in assistive devices and manipulators," in Proc. Joint Automat. Contr. Conf., 1980, vol. 1, paper TA-10-B

[6] N. Hogan, "Impedance control: An approach to manipulation," ASME J. Dyn. Syst., Measure. Contr., vol. 107, pp. 1-24, 1985. 
[7] M. H. Raibert and J. J. Craig, "Hybrid position/force control of manipulators," ASME J. Dyn. Syst., Measure. Contr., vol. 102, pp. 126-133, 1981.

[8] J. K. Salisbury, "Active stiffness control of a manipulator in Cartesian coordinates," in Proc. IEEE Conf. Decision Contr., 1980, pp. 95-100.

[9] S. A. Schneider and R. H. Cannon, "Object impedance control for cooperative manipulation: Theory and experimental results," IEEE Trans. Robot. Automat., vol. 8, pp. 383-394, 1992.

[10] D. Whitney, "Force feedback control of fine manipulator motions," ASME J. Dyn. Syst., Measure. Contr., vol. 2, pp. 91-97, 1977.

[11] R. Colbaugh, H. Seraji, and K. Glass, "Direct adaptive impedance control of robot manipulators," J. Robot. Syst., vol. 10, pp. 217-248, 1993.

[12] J. E. Colgate and N. Hogan, "Robust control of dynamically interacting systems," Int. J. Contr., vol. 48, pp. 65-88, 1988.

[13] H. Kazerooni, T. B. Sheridan, and P. K. Houpt, "Robust compliant motion for manipulators-Part 1: The fundamental concepts of compliant motion," IEEE Trans. Robot. Automat., vol. RA-2, pp. 83-92, 1986.

[14] H. Kazerooni, P. K. Houpt, and T. B. Sheridan, "Robust compliant motion for manipulators, Part 2: Design method," IEEE Trans. Robot. Automat., vol. RA-2, pp. 93-105, 1986.

[15] W. S. Newman, "Stability and performance limits of interaction controllers," ASME J. Dyn. Syst., Measure. Contr., vol. 114, pp. 563-570, 1992.

[16] R. W. Brockett and J. Loncarić, "The Geometry of Compliance Programming," in Theory and Applications of Nonlinear Control Systems C. I. Byrnes and A. Lindquist, Eds. Amsterdam, The Netherlands: North Holland, 1986, pp. 35-42

[17] J. Lončarić , "Geometrical analysis of compliant mechanisms in robotics," Ph.D. dissertation, Harvard University, Cambridge, MA 1985.

[18] "Normal forms of stiffness and compliance matrices," IEEE Trans. Robot. Automat., vol. RA-3, pp. 567-572, 1987.

[19] O. Khatib, "Real time obstacle avoidance for manipulators and mobile robots," Int. J. Robot. Res., vol. 5, pp. 90-99, 1986.

[20] D. E. Koditschek, "natural motion for robot arms," in Proc. IEEE Conf. Decision Contr., 1984, pp. 733-735.

[21] D. E. Koditschek, "The application of total energy as a Lyapunov function for mechanical control systems," Contemp. Math.: Dyn. Contr. Multibody Syst., vol. 97, pp. 131-157, 1989.

[22] A. J. van der Schaft, "Stabilization of Hamiltonian systems," Nonlinear Anal., Theory, Methods Applicat., vol. 10, pp. 1021-1035, 1986.

[23] M. Takegaki and S. Arimoto, "A new feedback method for dynamic control of manipulators," ASME J. Dyn. Syst., Measure. Contr., vol. 102, pp. 119-125, 1981.

[24] G. Meyer, "Design and global analysis of spacecraft attitude control systems," NASA Ames Res. Center, Tech. Rep. TR R-361, 1971

[25] T. Yoshikawa, Foundations of Robotics: Analysis and Control. Cambridge, MA: MIT Press, 1990.

[26] N. Hogan, "On the stability of manipulators performing contact tasks," IEEE Trans. Robot. Automat., vol. 4, pp. 677-686, 1988.

[27] H. Kazerooni, "Contact stability of the direct drive robot when constrained by a rigid environment," IEEE Trans. Automat. Contr., vol. 34, pp. 710-714, 1990.

[28] H. Kazerooni, B. Waibel, and S. Kim, "On the stability of compliant motion control: Theory and experiments," ASME J. Dyn. Syst., Measure. Contr., vol. 112, pp. 417-426, 1990.

[29] A. J. Bonnes and G. J. B. Colard, "Peg-in-hole assembly with the OSCAR -6 robot using impedance control," joint M.Sc. thesis reportnr. 021R94 , Univ. Twente, Enschede, The Netherlands, 1994.
[30] R. M. Hoogeveen, "A fast recognition and pose estimation of industrial objects (holle-Bol) using stereo vision," M.Sc. Thesis, Report 020M94, Univ. Twente, Enschede, The Netherlands, 1994.

[31] T. Tigchelaar, "Enhancement of the OSCAR -6 robot with vision," M.Sc thesis reportnr. 020R94, Enschede, The Netherlands, 1994

[32] H. van Brussel and J. Simons, "Automatic assembly by active force feedback accomodation," in Robot Sensors, Volume 2: Tactile and NonVision A. Pugh, Ed. Berlin: Springer, 1986, pp. 53-66.

[33] D. R. Strip, "Insertions using geometric analysis and hybrid forceposition control: Method and analysis," in Proc. IEEE Conf. Robot. Automat., vol. 3, pp. 1744-1751, 1988

[34] _ "A passive mechanism for insertion of convex pegs," in Proc. IEEE Conf. Robot. Automat., 1989, vol. 1, pp. 242-248.

[35] M. A. Peshkin, "Programmed compliance for error-corrective assembly," IEEE Trans. Robot. Automat., vol. 6, pp. 473-482, 1990.

[36] J. M. Schimmels and M. A. Peshkin, "Force assembly with friction," IEEE Trans. Robot. Automat., vol. 10, pp. 465-479, 1994.

[37] _ , "Admittance matrix design for force-guided assembly," IEEE Trans. Robot. Automat., vol. 8, pp. 213-227, 1992.

[38] S. P. Timoshenko and J. N. Goodier, Theory of Elasticity. New York: McGraw-Hill, 3rd ed., 1970

[39] D. R. J. Chillingworth, J. E. Marsden, and Y. H. Wan, "Symmetry and bifurcation in three-dimensional elasticity, Part 1," Arch. Rational Mechan. Anal., vol. 80, pp. 295-331, 1982.

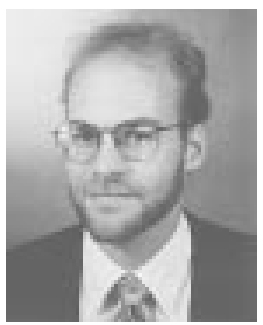

Ernest D. Fasse (M'90) received the S.B., S.M., and $\mathrm{Ph} . \mathrm{D}$. degrees in mechanical engineering from the Massachusetts Institute of Technology, Cambridge, in 1985, 1987, and 1992, respectively. His $\mathrm{Ph} . \mathrm{D}$. research was in human haptic interaction with mechanically simulated objects.

He was a post-Doctoral affiliate with the Control Laboratory of the Department of Electrical Engineering, University of Twente, The Netherlands, between September 1993 and August 1995. He is currently an Assistant Professor with the Department of Aerospace and Mechanical Engineering, University of Arizona. His research interests include robotics; energetic, human-machine interaction; and manufacturing.

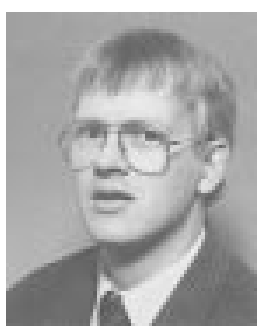

Jan F. Broenink (M'96) received the M.Sc. degree in electrical engineering and biomedical engineering in 1984 and the Ph.D. in electrical engineering in 1990 from the University of Twente, The Netherlands. His Ph.D. research was in the design of computer facilities for modeling and simulation of physical systems using bond graphs.

He is presently an Assistant Professor with the Control Laboratory of the Department of Electrical Engineering, University of Twente. His research interests include development of computer tools for modeling, simulation and implementation of embedded control systems; and robotics. 